Les ANNALES Les Annales de droit

DE DROIT

13 | 2019

Varia

\title{
La place du préfet de région dans l'organisation administrative française
}

The place of regional prefects in the French administrative organization

Yakouba Ouedraogo

\section{OpenEdition}

Journals

Édition électronique

URL : http://journals.openedition.org/add/1614

DOI : $10.4000 /$ add. 1614

ISSN : 2606-1988

Éditeur

Presses universitaires de Rouen et du Havre

Édition imprimée

Date de publication : 1 mai 2019

Pagination : 161-184

ISBN : 979-10-240-1293-3

ISSN : 1955-0855

Référence électronique

Yakouba Ouedraogo, "La place du préfet de région dans l'organisation administrative française », Les Annales de droit [En ligne], 13 | 2019, mis en ligne le 09 décembre 2019, consulté le 24 janvier 2021.

URL : http://journals.openedition.org/add/1614 ; DOI : https://doi.org/10.4000/add.1614 


\title{
La place du préfet de région dans l'organisation administrative française
}

\author{
Yakouba OuedRAogo
}

L'organisation administrative française est souvent présentée comme étant un «système préfectoral ${ }^{1} »$. En créant les préfets par la loi du 28 pluviôse an VIII, Napoléon a voulu faire de ces «empereurs aux petits pieds » des incarnations de l'État républicain déconcentré, de son ordre et de son autorité ${ }^{2}$. Le préfet est une autorité administrative dont le rôle est directement défini dans la Constitution du 4 octobre $1958^{3}$. Les nombreux tumultes que le préfet a traversés ${ }^{4}$, du fait notamment de la décentralisation territoriale, n'ont pas remis en cause la place qu'il occupe au sein des institutions administratives ${ }^{5}$.

1. Malik Boumediene, "La consolidation de la place du préfet dans l'administration déconcentrée de l'État", AJDA, 13 septembre 2004, n 30, 2004, p. 1644.

2. Bernard Larvaron, Le préfet face au $\mathrm{XXI}^{e}$ siècle, Paris, Economica, 2001, p. 73 ; Éric Kerrouche, Corps préfectoral et représentations de l'État, thèse, science politique, université Montesquieu-Bordeaux IV, 1997 ; Paul Bernard, Le préfet de la République. Le chêne et l'olivier, Paris, Economica, 1999.

3. Le préfet figure expressément parmi les hauts fonctionnaires qui sont nommés par décret délibéré en conseil des ministres, signé du Président de la République (art. 13 al. 3). L'art. 72 de la Constitution dispose également que: "Dans les collectivités territoriales de la République, le représentant de l'État, représentant chacun des membres du Gouvernement, a la charge des intérêts nationaux, du contrôle administratif et du respect des lois». Voir Roger Bonnaud-Delamare, «Le préfet dans la Constitution française de 1958 », R.I.S.A., 1961, p. 5-15.

4. Marc Ollivier, Conditions et compétences préfectorales, thèse, droit public, université Lumière-Lyon II, 3 décembre 2005, p. 10 et suiv.

5. Voir les deux rapports sénatoriaux récents consacrés au préfet: La marche de l'administration préfectorale, rapport d'information de $\mathrm{M}^{\mathrm{me}}$ Michèle André, fait au nom de la commission des finances, $\mathrm{n}^{\circ} 753$ (2013-2014), 17 juillet 2014; Les préfectures à l'heure de la réorganisation de l'administration territoriale de l'État (RéATE), Rapport d'information de $\mathrm{M}^{\mathrm{me}}$ Michèle André, fait au nom de la commission des finances du Sénat, $\mathrm{n}^{\circ} 77$ (2013-2014). 
Si le rôle du préfet est reconnu, sa fonction a longtemps été exercée à l'échelle départementale. Successeur de l'intendant de l'ancien régime ${ }^{6}$, le préfet de département devait être une courroie de transmission des décisions du pouvoir central à l'échelon local ${ }^{7}$.

La fonction préfectorale s'est toutefois éclatée depuis le milieu du $\mathrm{xx}^{\mathrm{e}}$ siècle. Au préfet de département se sont ajoutés le préfet de région et d'autres préfets sectoriels, comme le préfet maritime ${ }^{8}$ et le directeur de l'Agence régionale de santé, qualifié de "préfet sanitaire ${ }^{9}$ ». Ainsi, si «le préfet est ancien [...] la dualité entre préfet de département et préfet de région est récente. La différenciation date de $1964^{10}$ ». L'apparition du préfet de région s'est quelque peu faite «à la sauvette ${ }^{11}$ ». Après des évolutions dans sa dénomination ${ }^{12}$, sa fonction s'est stabilisée depuis le décret $\mathrm{n}^{0}$ 64-251 du 14 mars 1964 relatif à l'organisation des services de l'État dans les circonscriptions d'action régionale ${ }^{13}$. Depuis lors, le préfet de région tend à devenir un acteur administratif incontournable. Les réformes récentes confirment sa montée en puissance ${ }^{14}$.

La promotion de ce "nouveau préfet de région ${ }^{15}$ " interroge sur la place réelle qu'il occupe dans l'architecture administrative, c'està-dire son office, le statut qui lui est reconnu et les pouvoirs qui lui

6. Catherine Lecomte, «De l'intendant au préfet: rupture ou continuité ?», dans CURAPP, La loi du 28 pluviôse an VIII deux cents ans après, survivance ou continuité?, PUF, 2000, p. 11-26; Cédric Glineur, Histoire des institutions administratives. $X^{e}$-XIX $X^{e}$ siècle, Paris, Economica, 2017, p. 229 et 617.

7. Chaptal, archives parlementaires, séance du 28 pluviôse an VIII, cité par Philippe Gazagnes, «Préfet de région, préfet de département: dilution de l'autorité de l'État?», dans CURAPP, op. cit., p. 156.

8. Joseph Pini, «Considérations sur le préfet maritime», RFD adm., vol. 13, $\mathrm{n}^{\circ} 3$, mai-juin 1997, p. 559-580.

9. Jean-Michel de Forges, «Le volet hospitalier de la réforme de la protection sociale de $1996 », R D S S, \mathrm{n}^{\circ} 32,1996$, p. 713-742.

10. Jean-Marie Pontier, «Le nouveau préfet», $A J D A, \mathrm{n}^{\circ}{ }_{15}, 2010$, p. 819.

11. Philippe Gazagnes, art. cité, p. 160.

12. Préfet régional sous le régime de Vichy, commissaire de la République en 1944, Inspecteur général de l'administration en mission extraordinaire (IGAME) en 1948, préfet coordonnateur en 1959.

13. Olivier Gohin et Jean-Gabriel Sorbara, Institutions administratives, Paris, LGDJ, 2016 ; Nicolas Kada, Le préfet et la déconcentration sous la $\mathrm{V}^{e}$ République, thèse, droit public, université Grenoble II, 2000.

14. Bertrand Faure, Les institutions administratives, Paris, PUF, 2010, p. 189 et suiv.

15. Bertrand Landrieu, «Le nouveau préfet de région», Administration, $\mathrm{n}^{\circ} 205$, mars 2005, p. 12-14. 
permettent d'accomplir ses missions ${ }^{16}$. Se posent ainsi les questions de l'identification et de la nature de ses compétences, des relations qu'il doit entretenir avec le préfet de département qui lui a préexisté et auquel il tend à concurrencer, voire à supplanter. La montée en puissance du préfet de région entraîne-t-elle une substitution, une concurrence ou une complémentarité avec le préfet de département? Qu'en est-il du statut: l'unité de statut entre les deux types de préfets est-elle encore justifiée, ou faut-il évoluer vers une différenciation des statuts? Le positionnement du préfet de région dans l'architecture administrative suscite de nombreuses interrogations.

À travers de nombreuses réformes, le préfet de région connait une promotion dans ses fonctions et tend à devenir l'autorité territoriale de référence (1). Son statut comporte toutefois des incohérences, limitant ainsi son autonomisation dans l'architecture administrative (2).

\section{La promotion fonctionnelle du préfet de région}

Malgré son apparition récente, le préfet de région s'impose comme l'autorité territoriale de droit commun. C'est ce que témoignent l'accroissement de ses compétences (1.1) et l'affirmation de son autorité sur les autres agents déconcentrés, notamment les préfets de département (1.2).

\subsection{Une dynamique d'évolution des compétences}

Le préfet de région a connu une augmentation continue de ses compétences. Il est devenu l'autorité déconcentrée de droit commun.

\subsubsection{L'accroissement et l'approfondissement des compétences}

À l'origine de la régionalisation administrative, le préfet de région bénéficiait de compétences limitées, en rapport avec les pouvoirs restreints de la circonscription régionale, conçue à l'origine comme un espace d'intervention économique et d'aménagement du territoire ${ }^{17}$. Le décret du 14 mars 1964 lui a conféré un rôle de "technicien ${ }^{18}$ ", chargé de la coordination des administrations de l'État. Les réformes postérieures

16. Charles-Édouard Sénac, L'office du juge constitutionnel. Étude du contrôle de constitutionnalité par les juridictions françaises, Paris, LGDJ, 2015, t. 145, p. 28 et suiv.

17. Henri Oberdorff et Nicolas Kada, Les institutions administratives, Paris, Sirey, 2016.

18. Pascal Jan, Institutions administratives, Paris, Litec-LexisNexis, 2015, p. 20. 
ont étendu son champ d'intervention à de nouveaux domaines et lui ont reconnu de nouvelles attributions. Il est devenu, selon le décret du 29 avril 2004 modifié $^{19}$, le dépositaire de l'autorité de l'État, au même titre que le préfet de département ${ }^{20}$. L'article 2 du décret de 2004 en fait le "garant de la cohérence de l'action de l'État dans la région".

Le transfert de compétences au profit du préfet de région s'est opéré en plusieurs «actes ${ }^{21} »$. Un décret du 10 mai $1982^{22}$ l'a rebaptisé commissaire de la République de région et l'a fait délégué du gouvernement et représentant direct du Premier ministre et de chacun des ministres dans la région. Deux textes de $1992^{23}$ lui ont conféré un rôle de direction des activités des préfets de département de la région dans les domaines économique et social. Le décret du 29 avril 2004 a considérablement renforcé ses pouvoirs. Celui du 16 février 2010 portant réforme de l'administration territoriale de l'État (RéATE) ${ }^{24}$, édicté dans le cadre de la révision générale des politiques publiques (RGPP), consacre sa prééminence dans l'organisation territoriale ${ }^{25}$.

Le préfet de région bénéficie désormais d'une grande capacité d'initiatives, de propositions ou de décisions ${ }^{26}$. Depuis les réformes de 2004 et

19. D. $\mathrm{n}^{\circ} 2004-374,29$ avril 2004 modifié, relatif aux pouvoirs des préfets, à l'organisation et à l'action des services de l'État dans les régions et départements. Voir Francis Chauvin, «Le point sur... le décret $\mathrm{n}^{\circ} 2004-374$ du 29 avril 2004 relatif aux pouvoirs des préfets, à l'organisation et à l'action des services de l'État dans les régions et départements », RFAP, n ${ }^{\circ} 110,2004$, p. 394-399.

20. Art. 2 précité de la Constitution; art. $1^{\text {er }}$ al. 1 du décret du 29 avril 2004 modifié. Dominique Maillard Desgrées Du Loû, Institutions administratives, Paris, PUF, 2015, p. 479-480.

21. Pascal Jan, Institutions administratives, op. cit., p. 18-22.

22. Décret $n^{\circ} 82-390$ relatif aux pouvoirs des préfets de région, à l'action des services et organismes publics de l'État dans la région.

23. L. d'orientation $\mathrm{n}^{\circ} 92-125,6$ févr. 1992, relative à l'administration territoriale de la République dite ATR. Voir Maurice Bourjol, «La réforme de l'administration territoriale. Commentaire de la loi d'orientation du 6 février 1992 ", AJDA, 1992, $\mathrm{n}^{\mathrm{o}}$ spéc., p. 140-152. D. $\mathrm{n}^{\mathrm{o}}$ 92-604, $1^{\mathrm{er}}$ juill. 1992, portant charte de la déconcentration. Ce décret a définitivement consacré la région comme circonscription administrative.

24. Patrick Gérard, L'administration de l'État, Paris, LexisNexis, 2016, p. 215 et suiv.

25. Jean-Marie Pontier, art. cité, p. 819 et suiv. ; Pascal Combeau, « Les nouveaux visages territoriaux de la déconcentration ", RFD adm., $\mathrm{n}^{0}$ 5, 2010, p. 1011-1020; Geneviève Koubi, «Pouvoirs des préfets et cadres dirigeants de l'État. Une mise en perspective de la réforme de l'État», JCP adm., $n^{\circ} 9,1^{\text {er }}$ mars 2010, p. 35-38; Nicolas Kada et Isabelle Muller-Quoy, «Réforme de l'administration territoriale de l'État: les ratés de la RéATE», AJDA, 2011, p. 765-770; Johanne Saison-Demars, "La nouvelle répartition des pouvoirs au sein de la réforme de l'administration territoriale de l'État ", Revue Lamy des collectivités territoriales, $\mathrm{n}^{\mathrm{o}} 58,1^{\mathrm{er}}$ juin 2010, p. 45-46.

26. Olivier Gohin et Jean-Gabriel Sorbara, op. cit., p. 277. 
de 2010, il est devenu le représentant en chef de l'État au niveau local. Il préside le comité de l'administration régionale (CAR) et participe à la conférence nationale de l'administration territoriale de l'État ${ }^{27}$. Il conduit les politiques de développement économique et d'aménagement du territoire, négocie au nom de l'État les projets de contrats État-région, arrête après consultation du CAR le projet d'action stratégique de l'État dans la région ${ }^{28}$. Il détermine l'organisation fonctionnelle et territoriale des services déconcentrés des administrations placées sous son autorité. Il désigne le chef de projet chargé de l'animation et de la coordination de l'action des services intervenant dans la mise en œuvre d'une même politique.

Il exerce de nombreuses compétences de gestion administrative et financière. Il est responsable de l'exécution dans la région des politiques nationales et européennes ${ }^{29}$, de la gestion du patrimoine immobilier et des matériels des services de l'État placés sous son autorité ${ }^{30}$. Il arrête le schéma organisant la mutualisation des moyens entre les services de l'État. Il émet son avis sur les projets de budgets des services déconcentrés des administrations civiles de l'État. Il est l'autorité à laquelle les autorisations d'engagement et les crédits de paiement ouverts par la loi de finances au profit des services déconcentrés sont mis à disposition. Il arrête par programme la répartition des crédits alloués aux budgets opérationnels de programme. Il est ordonnateur secondaire des services déconcentrés ${ }^{31}$.

En matière de gestion du personnel, il est consulté par les autorités compétentes avant toute proposition de nomination, d'affectation ou de mutation des chefs de services déconcentrés et de leurs adjoints aux emplois de direction ou de chef de service déconcentré placé sous son autorité ainsi que de son adjoint. Depuis le décret $n^{0}$ 2015-1689 du 17 décembre 2015, il adresse également chaque année à l'autorité de nomination une évaluation sous forme d'appréciation littérale, ainsi qu'une proposition de notation pour chaque chef de service déconcentré placé sous son autorité. Il est informé de la note définitivement attribuée.

Il assure le contrôle administratif de la région, de ses établissements publics, des établissements publics interrégionaux qui ont leur siège

27. D. $\mathrm{n}^{\mathrm{o}} 2015-510,7$ mai 2015, art. 17, portant charte de la déconcentration.

28. Art. 5 du décret du 29 avril 2004 modifié.

29. Art. 2 du décret de 2004 modifié.

30. Art. 19 du décret de 2004 modifié.

31. Didier Maupas, «Le préfet de région et le nouveau cadre budgétaire : le point de vue d'un comptable public», Administration, $\mathrm{n}^{\circ}$ 205, 2005, p. 15-17. 
dans la région, ainsi que des établissements et organismes publics de l'État dont l'activité ne dépasse pas les limites de la région. Il veille à l'exercice régulier de leurs compétences par les autorités de la région ${ }^{32}$. Ces nombreuses compétences ressemblent à celles traditionnellement exercées par le préfet de département ${ }^{33}$.

L'accroissement des pouvoirs du préfet de région montre qu'il n'est plus seulement une autorité économique d'exécution, puisqu'il a acquis un pouvoir décisionnaire, et est érigé en autorité territoriale de droit commun.

\subsubsection{L'élévation au rang d'autorité déconcentrée de droit commun}

Le département a longtemps été considéré comme l'échelon général de la déconcentration territoriale ${ }^{34}$. L'inversion de la logique de répartition des compétences entre les administrations centrales et locales en $1992^{35}$ a ainsi profité au département. Le pouvoir de direction de la région, certes renforcé, restait cantonné à des matières spécifiques. Cette répartition des attributions a perduré jusqu'à la montée des circonscriptions régionales ${ }^{36}$. Celle-ci est liée à deux facteurs essentiels : d'une part la recherche d'un échelon dont la taille se prêterait mieux à un pilotage efficace des politiques publiques, et d'autre part l'influence européenne ${ }^{37}$. Les départements s'étant révélés inadaptés pour répondre à ces préoccupations ${ }^{38}$, c'est le niveau régional, situé «à l'épicentre d'un État unitaire», qui est apparu capable d'assurer «une synthèse

32. Art. 4 du décret du 24 avril 2004 modifié.

33. Loi 28 pluviôse an VIII. Art. 9, 10, 11 et 11-1 du décret du 29 avril 2004 modifié.

34. Marc Ollivier, op. cit., p. 90 et suiv.

35. L. $\mathrm{n}^{\circ}$ 92-125, 6 févr. 1992, relative à l'administration territoriale de la République dite ATR et décret du $1^{\mathrm{er}}$ juillet 1992 portant charte de la déconcentration.

36. Voir le $\mathrm{n}^{\circ} 196$ de la revue Administration, octobre 1998, consacré au thème de «l'État en région». Cour des comptes, L'organisation territoriale de l'État. Rapport public thématique, La Documentation française, juillet 2013, p. 63.

37. François Hulbert, Le pouvoir aux régions. La reconstruction géopolitique du territoire français, Paris, L'Harmattan, 2010, p. 131.

38. Hélène Pauliat et Clotilde Deffigier, «Le département peut-il encore rêver d'avenir ? ", RDP, $\mathrm{n}^{\circ}$ 5, 2015 dossier « Territoires : la réforme permanente », p. 1229-1240; Michel Verpeaux, "La fin des départements: chronique d'une mort annoncée», dans Mélanges en l'honneur du professeur Henri Oberdorff, Paris, LGDJ-Lextenso, 2015, p. 231-240; Aurélie Moriceau, "La réforme territoriale a-t-elle sauvé le département? ", Revue juridique de l'Ouest, $\mathrm{n}^{\circ}$ 1, janvier 2012, p. 21-45. 
des actions publiques ${ }^{39}$. De plus, l'«Europe des régions», apparue depuis le traité de Maastricht, fait de l'échelon régional l'interlocuteur privilégié des autorités européennes, notamment dans la gestion des fonds structurels.

La dynamique des régions a conduit à l'agrandissement de leur taille. La loi du 16 janvier 2015 relative à la délimitation des régions, qui a réduit leur nombre de 22 à 13 , se situe dans cette perspective ${ }^{40}$. Cette promotion concerne la région en tant que collectivité territoriale, mais elle influence la circonscription administrative régionale ${ }^{41}$. Bien que l'article 4 de la loi du 6 février 1992 prévoie que l'évolution des limites des collectivités territoriales est sans incidence sur les circonscriptions administratives de l'État ${ }^{42}$, les autorités ont jugé opportun d'adapter les services déconcentrés au nouveau découpage des régions. Le décret du 31 juillet 2015 fait ainsi coïncider le périmètre de certaines circonscriptions d'action régionale avec celui des nouvelles régions ${ }^{43}$.

Le décret du 29 avril 2004 a fait du préfet de région le garant de la cohérence, de l'animation et de la coordination de l'action des services de l'État et des préfets de département. La RéATE a confirmé cette promotion de la circonscription régionale ${ }^{44}$. Le conseil de modernisation des politiques publiques (CMPP) du 12 décembre 2007 a établi que «le niveau régional devient le niveau de droit commun pour mettre en œuvre les politiques publiques et piloter leur adaptation aux territoires».

39. Paul Bernard, "Le relais régional, base stratégique de l'État», Administration, $\mathrm{n}^{\circ} 179$, octobre 1998, p. 20 et 22. La Cour des comptes recommandait même dans un rapport thématique sur l'organisation territoriale de l'État de 2013, de « conforter la région comme échelon de référence» (Cour des comptes, L'organisation territoriale de l'État, op. cit., p. 204).

40. Patrick Gérard, op. cit., p. 215 ; Christophe Guettier, Institutions administratives, Paris, Dalloz, 2015.

41. Inspection générale de l'administration, Inspection générale des finances, Inspection générale des affaires sociales, L'évolution de l'organisation régionale de l'État consécutive à la nouvelle délimitation des régions, avril 2015.

42. On peut toutefois s'interroger sur la constitutionnalité de cet art. 4, car l'art. 72 de la Constitution parle de représentant de l'État, représentant de chacun des membres du Gouvernement dans les "collectivités territoriales de la République», ce qui semble en principe impliquer une coïncidence entre collectivités territoriales et circonscriptions administratives.

43. D. $\mathrm{n}^{\mathrm{o}} 2015-969$ du 31 juill. 2015 , modifiant le D. $\mathrm{n}^{\mathrm{o}} 60-516$ du 2 juin 1960 portant harmonisation des circonscriptions administratives, JORF, $\mathrm{n}^{\mathrm{o}}$ 0179, 5 août 2015 , p. 13415 .

44. Pascal Combeau, «Les nouveaux visages territoriaux... », art. cité, p. 1015. 
La circulaire du Premier ministre du 7 juillet 2008 confirme cette réorientation ${ }^{45}$. La tendance a été amplifiée par le décret du 16 février 2010 et la « RéATE II $^{46}$ ». La charte de la déconcentration issue du décret du 7 mai 2015 et la circulaire du 29 septembre 2015 réorganisant les secrétariats généraux pour les affaires régionales (SGAR) confortent la place d'autorité de droit commun du préfet de région. Elle reprend le principe selon lequel la région est la circonscription chargée, «sauf disposition législative contraire ou exception prévue par décret en conseil d'État», de l'animation et de la coordination des politiques nationales et de l'Union européenne ${ }^{47}$. La circonscription départementale, quant à elle, est devenue l'échelon de leur mise en œuvre. L'arrondissement reste la "porte d'entrée du réseau de l'administration de l'État ${ }^{48}$. Le préfet de région est corollairement devenu le pilote de droit commun des politiques nationales et européennes au niveau territorial. Le préfet de département intervient pour leur mise en œuvre. Le sous-préfet d'arrondissement est l'acteur chargé de l'animation du développement et de l'action locale ${ }^{49}$.

Certains services déconcentrés comme les agences régionales de santé (ARS) et les services régionaux de l'éducation, dirigés par les recteurs de région académique, échappent cependant à l'autorité du préfet de région. Suivant une forme originale de répartition des compétences ${ }^{50}$, les $\mathrm{ARS}^{51}$, établissements publics dotés de la personnalité juridique, sont soumises à l'autorité d'un directeur général ${ }^{52}$. Les organismes à caractère juridictionnel ou exerçant un contrôle des comptes, les services relevant du ministre de la Justice, les actions d'inspection de la législation du travail, ainsi que certains services chargés du paiement des dépenses publiques ou de la détermination de l'assiette et du recouvrement des

45. Circ. du 7 juillet 2008, relative à l'organisation de l'administration départementale de l'État, JORF, $\mathrm{n}^{\mathrm{o}} 0159$ du 9 juillet 2008, p. 11009.

46. La RéATE II désigne les mesures visant à adapter l'administration territoriale au nouveau découpage des régions effectué par la loi du 16 janvier 2015.

47. Art. 5 de la Charte de la déconcentration.

48. Les préfectures à l'heure de la réorganisation de l'administration territoriale de l'État (RéATE), op. cit., p. 7.

49. Art. 6 et 7 de la charte de la déconcentration du 7 mai 2015.

50. Pascal Combeau parle ainsi de « déconcentration verticale territorialisée » (art. cité, p. 1019).

51. L. $\mathrm{n}^{\mathrm{0}} 2009-879,21$ juillet 2009, portant réforme de l'hôpital et relative aux patients, à la santé et aux territoires (dite loi HPST) et D. $\mathrm{n}^{\mathrm{o}}$ 2010-336, 31 mars 2010, portant création des agences régionales de santé.

52. Jean-Michel de Forges, art. cité, p. 713-742. 
impôts et recettes publiques sont aussi soustraits à l'autorité du préfet de région ${ }^{53}$.

Le préfet de région intervient en qualité de "délégué territorial ${ }^{54}$ » à l'égard des établissements publics de l'État ayant un siège dans la région. Il coordonne les actions des établissements publics avec celles conduites par les administrations et les autres établissements publics de l'État ${ }^{55}$. À travers le CAR, l'ensemble des services déconcentrés et des établissements publics se trouve placé sous son pouvoir de coordination. Qualifié d' "état-major" de l'État régional ${ }^{56}$ ", le CAR réunit, sous la présidence du préfet de région les directeurs régionaux des administrations, le recteur de région académique et le directeur général de l'ARS ${ }^{57}$. Il assiste le préfet de région dans l'exercice de ses attributions en définissant les orientations stratégiques de l'État.

Le regroupement fonctionnel initié en 1982 a recentré un ensemble de services au niveau régional ${ }^{58}$. Les réformes ont promu une déconcentration horizontale au détriment de la déconcentration verticale. Après le regroupement des services en huit pôles régionaux ${ }^{59}$, la réforme de 2008 a procédé à la fusion de nombreux services déconcentrés pour donner huit directions régionales ${ }^{60}$. La réorganisation concerne également les $\mathrm{SGAR}^{61}$. Pour adapter les administrations de l'État à la nouvelle carte régionale, le rôle des SGAR a été redéfini et leur organisation restructurée $^{62}$. Dans les régions fusionnantes, sept SGAR préfigurateurs ont

53. Art. 2, 18, 32, 33 du décret du 29 avril 2004 modifié.

54. Art. 59-1 du décret du 29 avril 2004 modifié. Voir la liste des établissements publics concernés de l'État et les attributions du préfet de région dans le D. $\mathrm{n}^{\mathrm{o}}$ 2012-509, 18 avril 2012, JORF $\mathrm{n}^{0} 0094,20$ avril 2012, p. 7097, texte $\mathrm{n}^{\mathrm{o}} 4$.

55. L'exercice des compétences du préfet de région à l'égard des établissements publics soulève toutefois des difficultés d'application. Voir par exemple, CE, 20 février 2013, Fédération chimie énergie CFDT et autres, réq. $\mathrm{n}^{\mathrm{0}} 360307$. Hélène Pauliat, "Quels sont les pouvoirs des préfets sur les agences?", JCP Adm., $\mathrm{n}^{\mathrm{o}} 27,1^{\mathrm{er}}$ juillet 2013, p. $45-48$.

56. Circ. du 16 juin 2004, relative à l'application du décret du 29 avril 2004 modifié.

57. Art. 35 du décret du 29 avril 2004 modifié.

58. Wassim Kamel, «La nouvelle organisation de l'État en région», RFAP, 2010, vol. $136, \mathrm{n}^{\mathrm{o}} 4$, p. 1011-1022.

59. Décret du 5 octobre 2004.

60. Francis Chauvin, "La nouvelle administration régionale de l'État», AJDA, 2010, p. 825-830; Wassim Kamel, «La nouvelle organisation... », art. cité, p. 1017 et suiv.

61. Art. 8 du décret du 29 avril 2004 modifié, D. $n^{0} 2009-587$ du 25 mai 2009, relatif aux missions des secrétaires généraux pour les affaires régionales. Voir Jacques Fabre, «Le secrétaire général pour les affaires régionales », Administration, $\mathrm{n}^{\circ}$ 179, octobre 1998, p. 64-69.

62. Voir Circ. du 29 septembre 2015 relative à la Réforme territoriale de l'État - secrétariats généraux pour les affaires régionales. 
été désignés pour faciliter la mise en place de la nouvelle organisation de l'administration régionale ${ }^{63}$. L'adaptation des services régionaux de l'État élargit le champ de compétence du préfet de région, ses pouvoirs s'exerçant sur de grandes circonscriptions régionales.

En plus de ses compétences élargies, l'autorité des préfets de région est désormais reconnue sur les préfets de département et les chefs de services régionaux.

\subsection{Une autorité affirmée sur les agents et les services déconcentrés}

La principale innovation apportée par le décret du 16 février 2010 est l'affirmation de l'autorité du préfet de région sur le préfet de département et certains chefs de services régionaux. Cette innovation construit une unité de l'administration territoriale et établit une hiérarchie entre les représentants de l'État ${ }^{64}$. L'autorité du préfet de région est potentiellement très étendue. Sa nature demeure toutefois imprécise.

\subsubsection{L'étendue et les manifestations de l'autorité du préfet de région}

Jusqu'en 2010, la hiérarchie entre le préfet de région et le préfet de département était limitée aux attributions spécifiques du préfet de région ${ }^{65}$. Le décret du 16 février 2010 rompt désormais l'égalité entre ces deux représentants de l'État et confère au préfet de région «la stature d'un vrai "patron" de l'État territorial ${ }^{66}$. L'article $2.1 \mathrm{du}$ décret du 29 avril 2004 modifié prévoit expressément que le préfet de région "a autorité sur les préfets de département» et que cette autorité ne peut être déléguée. Selon le même article, «les préfets de département prennent leurs décisions conformément aux instructions que leur adresse le préfet de région. Le préfet de région peut également évoquer, par arrêté, et pour une durée limitée, tout ou partie d'une compétence à des fins de coordination régionale. Dans ce cas, il prend les décisions correspondantes en lieu et place des préfets de département». Les pouvoirs d'instruction et d'évocation constituent les modalités d'exercice de l'autorité du préfet de région.

63. Circ. du 29 septembre 2015, ann. 2.

64. Pascal Combeau, op. cit., p. 1013.

65. Mylène Le Roux, "Administrations déconcentrées", JurisClasseur Administratif, fasc. 116, à jour au 11 juillet 2012, spéc. $n^{\circ} 96$.

66. Patrick Gérard, op. cit., p. 215. 
Le pouvoir d'instruction consiste pour l'autorité supérieure à indiquer le comportement à adopter à l'autorité subordonnée. Le non-respect de l'instruction reçue constitue une faute disciplinaire exposant l'agent subordonné à des sanctions ${ }^{67}$. L'obligation pour le préfet de département de se conformer aux instructions émanant du préfet de région matérialise l'autorité de ce dernier ${ }^{68}$.

Le pouvoir d'évocation doit être justifié par un objectif d'intérêt régional et s'appliquer sur au moins deux départements de la région. Les nécessités de "coordination régionale», posées comme conditions à son exercice, deviennent un standard de répartition et d'exercice des compétences entre le préfet de région et le préfet de département.

Le pouvoir d'évocation du préfet de région se distingue du pouvoir d'évocation reconnu comme attribut du pouvoir hiérarchique ${ }^{69}$. Contrairement à ce dernier, il ne s'exerce pas sur des actes, mais vise des compétences. Il ne permet pas d'annuler ou de réformer les actes du subordonné, inexistants au moment de l'évocation. La circulaire du ministre de l'intérieur du 20 juillet 2010 relative à l'exercice du droit d'évocation par le préfet de région précise ainsi que «le droit d'évocation confère au préfet de région la capacité de modifier la répartition des compétences opérées par les normes réglementaires. Le préfet de région acquiert en quelque sorte la faculté d'élargir sa propre compétence dans les conditions définies par le décret ${ }^{70}$ ».

Malgré sa formulation en apparence générale, le pouvoir d'évocation comporte des exceptions ${ }^{71}$. Certaines matières relevant du domaine régalien restent la compétence exclusive du préfet de département: contrôle administratif du département, des communes, des établissements publics locaux et des établissements publics interdépartementaux, ainsi que des établissements et organismes publics de l'État; maintien

67. Art. 28 de la loi du 13 juillet 1983 portant droits et obligations des fonctionnaires. Voir Frédéric Colin, "De l'obligation pour tout agent public de respecter sa hiérarchie », AJFP, avril 2012, p. 92-95; Carole Moniolle, «La subordination dans la fonction publique», $A J D A, 13$ septembre 2010, p. 1630.

68. Jean-Michel Bricault, «Régionalisation de l'administration déconcentrée et question du maintien de la règle du dédoublement fonctionnel du préfet de région », $R R J, \mathrm{n}^{\circ} 4,2009$, p. 1991.

69. Mathieu Houser, «Le droit d'évocation du préfet de région », Revue administrative, $\mathrm{n}^{\mathrm{o}} 378,2010$, p. 584-589.

70. Voir le tableau des exemples de domaines pouvant faire l'objet du droit d'évocation en annexe de la circulaire.

71. Art. 2.1. du décret du 29 avril 2004 modifié. 
de l'ordre public et de la sécurité des populations; entrée et séjour des étrangers et du droit d'asile ${ }^{72}$.

Le préfet de région a également autorité sur les chefs des services déconcentrés, les délégués ou les correspondants à l'échelon régional des administrations civiles de l'État ${ }^{73}$ et sur les chefs des administrations civiles de l'État dont l'action s'étend au-delà de la région ou présente, en tout ou partie, un caractère interrégional ${ }^{74}$. Pour ce dernier type de services, il a "autorité fonctionnelle » sur le chef de service, pour la part de son activité qui s'exerce dans les limites de la région ${ }^{75}$.

Une exception notable à l'autorité du préfet de région concerne le directeur général de l'ARS. Le préfet de région exerce ses compétences "sous réserve des compétences de l'agence régionale de santé ${ }^{76}$ ». Le directeur de l'ARS participe néanmoins en tant que membre du CAR à la définition des orientations stratégiques de l'État en région.

Malgré son affirmation, la nature de l'autorité du préfet de région reste encore imprécise.

\subsubsection{Le refus d'un pouvoir hiérarchique et la consécration d'une hiérarchie fonctionnelle du préfet de région}

La signification du terme "autorité », employé par le décret de 2004 modifié, et sa nature, méritent des éclaircissements. L'autorité peut reposer sur une condition factuelle ou sur une norme juridique. Dans le premier cas, elle désigne l'influence qu'une personne peut avoir sur une autre personne ou sur un groupe, lui permettant de s'imposer.

72. Art. 11-1 du décret du 29 avril 2004 modifié.

73. Art. 17 du décret du 29 avril 2004 modifié.

74. Art. 18 du décret du 29 avril 2004 modifié.

75. L'«autorité fonctionnelle» est également reconnue au préfet de département sur les chefs de service des administrations civiles, dont l'activité s'étend au-delà du département et présente, en tout ou partie, un caractère interdépartemental (art. 18).

76. Art. 2 du décret de 2004 modifié. Voir Philippe Ligneau, «Les régions, nouveaux acteurs dans le secteur social et de la santé», RDSS, 1996, p. 485-501; Marie-Laure Moquet-Anger, "Les aspects de la nouvelle organisation territoriale de la santé ", JCP Adm., $\mathrm{n}^{\circ}$ 39, étude 2224, septembre 2009, p. 20-25; Marie-Laure MoquetAnger, "La régionalisation du système de santé », dans Les collectivités territoriales. Mélanges en l'honneur de Jacques Moreau, Paris, Economica, 2003, p. 313-323. 
En droit public ${ }^{77}$, le terme autorité est, certes, comme le relève JeanMarie Pontier, «très répandu [...], mais il n'est pas d'une signification évidente ${ }^{78}$ ». Il est susceptible de plusieurs définitions ${ }^{79}$. Dans une dimension substantielle, l'autorité s'assimile au pouvoir reconnu pour l'exercice d'une fonction. Elle est synonyme dans ce cas de pouvoir de décision, de faculté de commandement et de direction ${ }^{80}$. Dans une dimension institutionnelle, l'autorité désigne une personne ou un organe légalement investi d'un pouvoir de décision ou de commandement, incarnant par lui-même un tel pouvoir. Dans ce sens, on parle souvent de "personne dépositaire de l'autorité publique ${ }^{81}$ ", d'autorité compétente, d'autorité judiciaire ${ }^{82}$ ou d'autorité administrative ${ }^{83}$ (indépendante, le cas échéant). L'autorité peut également désigner la qualité ou la force juridique attachée à certains actes ou sources du droit. Ainsi parle-t-on d'autorité de la chose jugée, de l'autorité de la loi ${ }^{84}$ ou encore de l'autorité des traités internationaux régulièrement ratifiés ou approuvés sur celle des lois ${ }^{85}$ ou, dans la doctrine, d'un auteur qui fait autorité. Dans une approche fonctionnelle enfin, l'autorité sert, dans les institutions publiques et privées, de mode de structuration et de régulation des relations entre leurs membres. Elle désigne ce «lien hiérarchique" conférant une ascendance à l'agent supérieur sur ses subordonnés. L'autorité du préfet de région sur les préfets de département et les chefs de services déconcentrés s'envisage selon ce sens fonctionnel.

Se pose ensuite la question de la nature de l'autorité reconnue au préfet de région. S'agit-il d'un pouvoir hiérarchique? Le décret de 2004 modifié n'utilise pas cette expression, mais s'agissant d'agents publics, le

77. Le terme autorité est également utilisé en droit privé. En droit de la famille par exemple, l'autorité parentale désigne un ensemble de droits et de devoirs reconnus aux parents et ayant pour finalité l'intérêt de l'enfant (C. civ., art. 371-1).

78. Jean-Marie Pontier, art. cité, p. 821.

79. Soazick Kerneis, "Autorité», dans Denis Alland et Stéphane Rials (dir.), Dictionnaire de la culture juridique, Paris, PUF, 2003, p. 111 ; Bruno Domingo, "Autorité », dans Nicolas Kada et Martial Mathieu (dir.), Dictionnaire d'administration publique, Grenoble, PUG, 2014, p. 44.

80. C'est dans ce sens qu'il faut l'entendre, lorsqu'on parle de crise de l'autorité, ou encore de l'«abus d'autorité » puni par le droit (C. pén., art. 432-1).

81. C. pén., art. $432-1$ et suiv.

82. Titre VIII de la Constitution du 4 octobre 1958.

83. Le préfet relève de cette catégorie d'autorités.

84. Titre III de la Constitution de 1791 : «Il n'y a point en France d'autorité supérieure à celle des lois. Le roi ne règne que par elle, et ce n'est qu'au nom de la loi qu'il peut exiger l'obéissance."

85. Art. 55 de la Constitution du 4 octobre 1958. 
lien d'autorité s'exprime généralement par le pouvoir hiérarchique ${ }^{86}$. Ce rapprochement est d'autant plus logique que «l'attribution d'un pouvoir hiérarchique peut apparaître comme un élément décisif au fondement d'une autorité ${ }^{87}$ ".

L'incertitude sur la nature du lien entre le préfet de région et les préfets de département et les chefs de services déconcentrés s'observe dans la doctrine et dans la jurisprudence. Les auteurs retiennent les qualifications de "supériorité hiérarchique ", de "pouvoir quasi hiérarchique ", et même de "pouvoir hiérarchique ${ }^{88}$ ». Le Conseil d'État a considéré dans son arrêt du 20 février 2013, Fédération chimie énergie CFDT et autres, que les dispositions du décret du 18 avril 2012, attribuant au préfet un rôle de coordination des actions de certains établissements publics de l'État et de représentation dans la région ou le département, lui « confèrent $[\ldots]$ un pouvoir hiérarchique au sein de l'établissement et ont ainsi le caractère de règles constitutives ${ }^{89}$ ".

La nature incertaine de l'autorité du préfet de région oblige à la caractériser par rapport au pouvoir hiérarchique ${ }^{90}$. Si le pouvoir hiérarchique est lui aussi imprécis ${ }^{91}$, il consiste fondamentalement, selon Marcel Prélot, « dans la pleine et entière autorité du chef sur ses subordonnés en tout ce qui regarde le service public ${ }^{92} »$. Il comprend deux catégories de compétences, qui s'exercent sur la personne du subordonné et à l'égard de ses actes ${ }^{93}$. Le pourvoir sur la personne du subordonné implique

86. Pierre Di Malta, Essai sur la notion de pouvoir hiérarchique, Paris, LGDJ, 1961; Clément Chauvet, Le pouvoir hiérarchique, Paris, LGDJ-Lextenso, 2013.

87. Corinne Charlery, Les services déconcentrés et la modernisation de l'État, thèse de droit public, Paris I, 1997 ; Carole Moniolle, « La subordination... », art. cité, p. 1630.

88. Pascal Combeau, "Les nouveaux visages territoriaux de la déconcentration», op. cit., p. 1014 ; Bertrand Faure, Les institutions administratives, op. cit., p. 188 et 189; Christophe Guettier, op. cit., p. 428.

89. CE, 20 février 2013, Fédération chimie énergie CFDT et autres, arrêt cité. Voir Hélènen Pauliat, art. cité, p. 45-48.

90. Pierre Serrand, Manuel d'institutions administratives françaises, Paris, PUF, 2015, p. 167.

91. André Legrand, «Un instrument flou : le pouvoir hiérarchique», dans Mélanges en l'hommage à Roland Drago, Economica, 1996, p. 59-78; Jean Rivero, «Remarques à propos du pouvoir hiérarchique», $A J D A, 1966$, p. 154; Jean-Claude Groshens, «À propos du pouvoir hiérarchique dans l'administration. Le pouvoir des supérieurs hiérarchiques sur les actes de leurs subordonnés», AJDA, mars 1966, p. 141-153; Paul-Marie Gaudemet, «Le déclin de l'autorité hiérarchique », D., 1947, chron., p. 137.

92. Marcel Prélot, Cours de droit administratif, Licence $2{ }^{\mathrm{e}}$ année, 1948-1949, cité dans Pierre Di Malta, op. cit., p. 1.

93. Nadine Poulet-Gibot Leclerc, «Le pouvoir hiérarchique», RFDA, mai-juin 2007, p. 508 et suiv.; Pierre Di Malta, op. cit., p. 75. 
le pouvoir de nomination et d'affectation, le pouvoir de notation et le pouvoir disciplinaire ou de sanction. Le pouvoir sur les actes comprend un pouvoir de contrôle - conférant au supérieur une compétence d'annulation et une compétence de réformation des actes du subordonné et un pouvoir d'instruction, par lequel le supérieur fixe les mesures d'organisation du service et détermine le comportement attendu de ses subordonnés, tenus par un devoir d'obéissance ${ }^{94}$.

Le préfet de région ne dispose pas de l'ensemble de ces prérogatives sur la personne et les actes des préfets de département et des chefs des services déconcentrés. En matière d'emploi, le préfet de département est nommé, tout comme le préfet de région, par décret en conseil des ministres signé par le Président de la République. Les directeurs des administrations régionales sont nommés par arrêté du ou des ministres dont relève la direction régionale, après avis du préfet de région ${ }^{95}$. En matière de notation des chefs des services déconcentrés, il dispose d'un simple pouvoir de proposition, mais il est informé de la note définitivement attribuée à l'agent ${ }^{96}$. Concernant le pouvoir sur les actes, le préfet de région ne bénéficie que partiellement des attributions d'un pouvoir hiérarchique. Les pouvoirs d'annulation et de modification ne lui sont pas reconnus. Les recours hiérarchiques contre les décisions du préfet de département et du préfet de région sont adressés aux ministres compétents $^{97}$. Seuls le pouvoir d'instruction et le droit d'évocation sont expressément prévus.

Or, le droit d'évocation n'est ni une compétence d'annulation ni une compétence de réformation des actes. Il peut être exercé en l'absence même de décision du préfet de département ${ }^{98}$. L'emploi du terme «droit " par le décret, au lieu de celui de " pouvoir », traduit le refus de le rapprocher du pouvoir d'évocation reconnu au supérieur hiérarchique. Selon l'instruction du 20 juillet 2010, «le droit d'évocation dévolu au préfet de région est ainsi distinct du pouvoir d'évocation comme attribut

94. Frédéric Colin, «De l'obligation pour tout agent public de respecter sa hiérarchie», op. cit., p. 92-95.

95. D. $\mathrm{n}^{\circ}$ 2009-360, 31 mars 2009 art. 10 relatif aux emplois de direction de l'administration territoriale de l'État; art. 30 du décret du 29 avril 2004 modifié.

96. Art. 31 du décret du 29 avril 2004 modifié.

97. Art. 2.2 du décret du 29 avril 2004. Voir également la circulaire $n^{0}$ 5506/SG du Premier ministre du 13 décembre 2010, portant application du décret du 16 février 2010. Voir Tatiana Gründler, «La réforme de l'État territorial vue à travers le prisme de la circulaire du 13 décembre 2010, ou l'influence des enjeux économiques sur l'organisation du territoire", Gestion et finances publiques, $\mathrm{n}^{\circ} 11$, novembre 2011, p. $800-803$.

98. Mathieu Houser, art. cité, p. 588. 
du pouvoir hiérarchique permettant d'annuler et de réformer les actes d'un subordonné ». Il s'agit d'une "catégorie sui generis ${ }^{99}$ ", distincte du pouvoir d'évocation reconnu dans le cadre du pouvoir hiérarchique, qui permet au préfet de région, sous le motif de la coordination régionale, de se substituer ex ante au préfet de département. L'exercice de ce droit entraîne le dessaisissement du préfet de département.

L'autorité du préfet de région est loin d'être un véritable pouvoir hiérarchique. Il lui manque les pouvoirs de nomination, d'affectation, de sanction, ainsi que la compétence d'annulation et de réformation des actes. L'appartenance du préfet de région et du préfet de département à un même corps rend difficile l'exercice d'un véritable pouvoir hiérarchique. L'autorité du préfet de région lui confère un simple ascendant fonctionnel sur les préfets de département et les chefs de services déconcentrés.

L'absence d'un pouvoir hiérarchique résulte du décret du 29 avril 2004 et de la circulaire du 16 juin 2004 relative à son application ${ }^{100}$. Le troisième CMPP du 11 juin 2008 a aussi retenu une formule non moins ambiguë, selon laquelle l'autorité du préfet de région dans le pilotage des politiques publiques «se traduit, en particulier, par son autorité hiérarchique sur le préfet de département au travers, notamment, d'un pouvoir d'évocation ». Les pouvoirs publics ont voulu ménager les susceptibilités des préfets de département, en retenant une formule qui «va au-delà d'un simple ascendant fonctionnel, sans aller jusqu'à la supériorité hiérarchique, car le préfet de région reste aussi préfet de département ${ }^{101}$ ». Le ministre reste ainsi le véritable supérieur hiérarchique du préfet de département et des chefs des services déconcentrés ${ }^{102}$.

Selon Jean-Marie Pontier, l'autorité dont bénéficie le préfet de région pourrait être considérée comme une nouvelle forme de pouvoir hiérarchique ${ }^{103}$. Si l'on admet, comme Charles Eisenmann, qu'il y a des "pouvoirs hiérarchiques», et que chacune de ces prérogatives peut exister isolément, l'autorité du préfet de région pourrait être qualifiée

99. Ibid., p. 586.

100. La circulaire prévoyait explicitement que «le préfet de région ne dispose pas d'un pouvoir hiérarchique sur les préfets de département, afin de ne pas diluer les responsabilités et d'éviter d'alourdir le processus de décision déconcentré ».

101. Henri Oberdorff et Nicolas Kada, op. cit., p. 162.

102. Philippe Cossaltier, «Le pouvoir hiérarchique des ministres sur les agences régionales de santé», Revue générale du droit, $\mathrm{n}^{\mathrm{o}} 2,1^{\mathrm{er}}$ janvier 2013, http://www.revuegeneraledudroit.eu/?p=4317.

103. Jean-Marie Pontier, art. cité, p. 823. 
de «pouvoir hiérarchique incomplet, partiel, limité ${ }^{104}$ ». On pourrait finalement la qualifier de «hiérarchie fonctionnelle ${ }^{105}$ ».

Malgré l'autorité dont bénéficie le préfet de région sur le préfet de département, il reste soumis au même statut que ce dernier.

\section{Les ambiguïtés du statut du préfet de région}

Le statut désigne un ensemble de règles législatives ou réglementaires régissant les différents aspects de la condition juridique des agents publics ${ }^{106}$, en tant qu'ils appartiennent à la catégorie générale des fonctionnaires ou à une catégorie particulière ${ }^{107}$. Le corps des préfets est l'un des statuts particuliers les plus prestigieux de la fonction publique ${ }^{108}$. Il fait partie des emplois supérieurs auxquels les nominations sont laissées à la décision du Gouvernement ${ }^{109}$. L'accroissement des compétences du préfet de région et l'affirmation de son autorité sur les préfets de département et les chefs de services déconcentrés devaient entraîner une adaptation de son statut à la nouvelle place qu'il occupe dans l'administration déconcentrée de l'État ${ }^{110}$. Malgré cette montée en puissance, l'identité de statut avec le préfet de département a été maintenue. La cohérence des réformes administratives soulève ainsi la question de la pertinence du maintien de ce dédoublement fonctionnel (2.1) et celle de l'unité du statut (2.2).

104. Charles Eisenmann, Cours de droit administratif, Paris, LGDJ, 1982, p. 240, cité par Mathieu Houser, op. cit., p. 585.

105. Le concept d'«autorité fonctionnelle» est d'ailleurs employé par le décret modifié à propos des services dont l'action présente un caractère interrégional.

106. L'entrée au service, les droits et obligations, la carrière, la discipline, la sortie du service.

107. Gérard Cornu (dir.), Vocabulaire juridique, Paris, PUF, 2016, p. 990.

108. D. $\mathrm{n}^{\mathrm{0}} 64-805,29$ juill. 1964 modifié, art. $1^{\text {er }}$ al. 1 , fixant les dispositions réglementaires applicables aux préfets.

109. L. $\mathrm{n}^{\mathrm{0}} 84-16,11$ janv. 1984 , art. 25 , portant dispositions statutaires relatives à la fonction publique de l'État. D. $\mathrm{n}^{\mathrm{o}} 85-779,24$ juill. 1985 , art. $1^{\mathrm{er}}$, portant application de l'art. 25 de la L. $n^{\circ} 84-16,11$ janv. 1984.

110. Jean-Michel Bricault, "Montée en puissance du préfet de région: se poser la question du maintien de son dédoublement fonctionnel ", Administration, $\mathrm{n}^{\circ}{ }_{226}$, juin-juillet 2010, p. 122-127. 


\subsection{La question de la pertinence du dédoublement fonctionnel}

Le dédoublement fonctionnel ne s'entend pas ici dans le sens qui lui est ordinairement attribué. Il désigne généralement la situation de l'agent public qui remplit des fonctions relevant de deux ou plusieurs ordres de compétences et se trouve ainsi soumis à des hiérarchies et à des contrôles de nature différente. La situation du maire constitue une parfaite illustration, puisqu'il cumule ses attributions d'organe exécutif de collectivité territoriale (la commune) - le plaçant sous le contrôle administratif du préfet de département - avec celles d'autorité administrative, exercées sous le contrôle hiérarchique du préfet. Il participe même, en tant qu'officier d'état civil et de police judiciaire, à des activités d'ordre judiciaire placées sous l'autorité du procureur de la République et des tribunaux ${ }^{111}$. Le préfet se trouvait dans la même situation de dédoublement fonctionnel avant la loi du 2 mars 1982 modifiée, car en plus de sa qualité d'autorité déconcentrée, il exerçait les fonctions d'organe exécutif de la région ou du département collectivité territoriale. La réforme de 1982 a supprimé ses compétences d'organe exécutif local. Seule la compétence de contrôle administratif des actes des collectivités régionales et départementales et de leurs établissements publics subsiste.

Le dédoublement fonctionnel dont il s'agit ici est différent de celui qui existait avant 1982. Il s'entend de la double casquette du préfet de région, qui est à la fois représentant de l'État dans la région et dans un des départements de la région. Il s'agit d'un dédoublement dans la fonction préfectorale. Aux termes de l'article 7 du décret du 29 avril 2004 modifié, «le préfet de région est le préfet du département où se trouve le chef-lieu de la région». Selon Philippe Gazagnes, cette disposition est «la clé du dispositif et l'assurance de l'absence de double échelon ${ }^{112}$ » entre les deux types de préfets, alors que la montée en puissance du préfet de région tend à dissocier ses fonctions de celles du préfet de département. L'auteur en conclut que la qualité de " "préfet de région" est et reste avant tout un titre». Le maintien du dédoublement fonctionnel du préfet de région se justifiait par certains avantages que cette organisation comporterait. Mais il apparaît de plus en plus impertinent au regard de la situation actuelle du préfet de région.

111. Art. 16 et 19 du Code de procédure pénale, art. L. 2122-31 du Code général des collectivités territoriales.

112. Philippe Gazagnes, art. cité, p. 160. 


\subsubsection{Les avantages supposés du dédoublement fonctionnel}

Malgré la montée en puissance du préfet de région, les réformes successives se sont gardées de mettre fin au dédoublement fonctionnel. Plusieurs considérations ont justifié le choix des pouvoirs publics de ne pas consacrer une fonction spécifique de préfet de région. La première et plus importante raison est politique. Le maintien du dédoublement fonctionnel vise à ménager les susceptibilités des préfets de département et à éviter ainsi de créer des tensions au sein de ce corps ${ }^{113}$. Consacrer des préfets de région personnellement et statutairement distincts des préfets de département pourrait engendrer la méfiance des seconds à l'égard de leurs collègues régionaux, d'autant plus que ces derniers administrent des circonscriptions géographiquement plus étendues. La crainte des préfets de département à l'égard de la dissociation des deux fonctions est le risque d'une trop grande subordination aux préfets de région. C'est ce qui explique la lente émergence d'une autorité de l'échelon régional sur le niveau départemental. Conjuguée à l'autorité du préfet de région, la dissociation des deux fonctions participerait de la «souspréfectorisation » ou de la "super sous-préfectorisation ${ }^{114}$ » des préfets de département. Or, le dédoublement fonctionnel évite l'éclatement des deux fonctions.

Les raisons du maintien du dédoublement fonctionnel sont ensuite d'ordre technique. Il s'agit d'éviter la multiplication des statuts en créant un corps supplémentaire au sein de l'administration. Sur le plan financier, la séparation des deux fonctions serait une « fausse bonne idée : elle rendrait plus difficile la pratique des regroupements fonctionnels qui consiste, dans une administration déconcentrée, à placer sous une même autorité la direction régionale et la direction départementale implantée au chef-lieu de région en vue d'optimiser l'emploi des moyens ${ }^{115}$ ». Le maintien du dédoublement fonctionnel permettrait au contraire de réaliser des économies d'échelle.

$\mathrm{Du}$ point de vue pratique, le dédoublement fonctionnel permet au préfet de région de ne pas se couper de la base et de rester plus près des administrés. La crainte d'une déconnexion avec le niveau opérationnel a été exprimée par le sénateur Jean-Pierre Sueur lors des débats au Sénat sur la loi $\mathrm{n}^{\mathrm{o}} 2004-809 \mathrm{du} 13$ août 2004, relative aux libertés et responsabilités locales. Il redoutait, «en créant un préfet de région

113. Jean-Marie Pontier, art. cité, p. 823.

114. La marche de l'administration préfectorale, op. cit., p. 89 ; Maurice Bourjol, art. cité, p. 141.

115. Francis Chauvin, «Le point sur... le décret $n^{\circ}$ 2004-374...», art. cité, p. 398. 
qui ne serait pas préfet de département, qu'on en fasse un secrétaire général aux affaires régionales", qui n'aurait "pas autant de liens avec les réalités du terrain que les préfets» de département ${ }^{116}$. En clair, en étant également préfet de département, le préfet de région adopte " une pratique administrative comparable à celle de ses collègues et peut donc développer avec crédibilité un ascendant fonctionnel sur les autres préfets de département ${ }^{117}$ ». De ce fait, «il y a [...] moins de risques politiques découlant de prérogatives excessives pour une autorité territoriale ${ }^{118}$.»

Malgré ces avantages, le dédoublement fonctionnel semble de plus en plus incohérent au regard de la montée en puissance du rôle du préfet de région.

\subsubsection{Les incohérences du dédoublement fonctionnel et les justifications d'une disjonction des deux fonctions}

Si le dédoublement des fonctions de préfet de région et de préfet de département chef-lieu de la région est jusqu'à présent maintenu, ce n'est sans doute pas pour les avantages et les raisons qui l'ont justifié au départ. Le cumul des deux fonctions semble désormais présenter plus d'inconvénients que d'avantages. Comme le souligne Jean-Marie Pontier « les raisons de 1964 ont disparu en 2010, l'institution régionale est suffisamment installée, la période de rodage est depuis longtemps terminée, les avantages du cumul des fonctions ne sont plus suffisants pour refuser de passer à l'étape suivante ${ }^{119}$ ". La dissociation des deux fonctions permettrait de faire du préfet de région une sorte de «super préfet », personnellement distinct du préfet de département. Plusieurs raisons montrent que le dédoublement fonctionnel ne semble plus correspondre à la situation du préfet de région dans l'architecture administrative.

On peut en premier lieu relever l'accroissement des compétences du préfet de région. L'étendue des compétences du préfet de région et leurs natures variées militent en faveur de l'institution d'un préfet de plein exercice. Avec la montée en puissance de la région, désormais

116. Cité par Jean-Michel Bricault, «Régionalisation de l'administration déconcentrée... », art. cité, p. 1998, spéc. Note 28.

117. Henri Oberdorff et Nicolas Kada, op. cit., p. 161.

118. Ibid.

119. Jean-Marie Pontier, art. cité, p. 823. 
circonscription déconcentrée de principe, le préfet de région est subséquemment devenu l'autorité territoriale de droit commun, à vocation générale et interministérielle.

En deuxième lieu, la disjonction des deux fonctions serait un gage d'allègement des tâches de préfet de département du chef-lieu de la région. Le cumul des deux fonctions entraîne une surcharge de travail pour cette autorité. Les réformes territoriales actuelles, tendant à adapter les circonscriptions régionales aux périmètres des grandes régions issues du découpage de la loi du 16 janvier 2015, militent davantage en faveur de l'abandon du dédoublement fonctionnel. La réduction du nombre des circonscriptions régionales entraînerait un agrandissement de leur taille et de celle des services déconcentrés de l'État. Il serait logique que l'élargissement des circonscriptions régionales soit accompagné par l'institution d'un préfet de région exclusivement chargé de la représentation de l'État et de la défense des intérêts nationaux dans ces grandes circonscriptions.

En troisième lieu, le partage des tâches et des responsabilités qui incombent aux préfets du fait de l'exercice des deux fonctions commande de mettre fin au dédoublement fonctionnel. Dès 2000, le rapport Mauroy, Refonder l'action publique locale: rapport au Premier ministre, préconisait de «décharger [le préfet de région de ses] tâches départementales pour être l'animateur véritable des services régionaux et le correspondant des programmes interrégionaux », en « le distinguant des préfets des départements, chefs-lieux de région ${ }^{120}{ }$. Lors de l’adoption de la loi du 13 août 2004 précitée, le sénateur Pierre Mauroy faisait remarquer que le fait «d'avoir un préfet de région qui soit en même temps préfet de département n'est pas sans inconvénient [...]. Dès que les régions prendront leur véritable dimension, nous aurons besoin de préfets de région qui ne se consacrent qu'à cette mission ${ }^{121}$ ». L'autonomisation des deux autorités permettrait ainsi d'améliorer la lisibilité du processus de régionalisation administrative, en dotant la circonscription régionale d'une autorité pleinement chargée d'y défendre les intérêts de l'État.

Enfin, la déconnexion entre les deux fonctions paraît être l'aboutissement logique de l'orientation des réformes administratives menées

120. Refonder l'action publique locale : rapport au Premier ministre Pierre Mauroy, Paris, La Documentation française, «Rapports officiels", janvier 2000, p. 80, proposition $n^{\circ} 63$.

121. Jean-Michel Bricault, "Régionalisation de l'administration déconcentrée...», art. cité, p. 1997-1998. 
depuis 1982, et surtout depuis l'adoption du décret du 29 avril 2004 et la RéATE. Ces réformes ont accentué la distinction entre les deux types de préfets, selon un double sens de hiérarchisation-spécialisation: hiérarchisation du fait de l'affirmation de l'autorité du préfet de région sur le préfet de département, mettant fin à l'égalité fonctionnelle entre les deux agents, et spécialisation parce que la conception du rôle et des compétences de chacune de ces autorités a évolué. L'élévation du préfet de région au rang d'autorité déconcentrée de droit commun, supérieur fonctionnel du préfet de département, et la réorientation des fonctions de ce dernier vers des actions de mise en œuvre opérationnelle des politiques publiques, selon une logique de proximité ${ }^{122}$, sont la preuve de la dissociation des deux fonctions préfectorales.

Dans cette structure administrative réorganisée, c'est le préfet de région qui est la figure centrale de l'administration déconcentrée. Plus récent dans le paysage administratif, il s'identifie facilement à la représentation interministérielle ${ }^{123}$, contrairement au préfet de département dont la mission prioritaire de maintien de l'ordre public conforte l'image de représentant du ministre de l'Intérieur, au titre duquel les deux types de préfets sont pourtant nommés ${ }^{124}$. Les compétences du préfet de département sont subordonnées à l'autorité et au pouvoir de coordination du préfet de région dans de nombreux domaines stratégiques. Son autonomie d'action n'est préservée qu'en matière de politiques de proximité.

Dans les autres cas, il exerce ses compétences «sous réserve» de l'intervention du préfet de région. Il paraît ainsi évident que « la fonction de préfet de région est devenue largement prééminente en termes de commandement stratégique et celle de préfet de département apparaît de plus en plus comme une fonction de gestion et d'exécution opérationnelle. De cette différenciation ne découle-t-il pas une incohérence de fond à maintenir au préfet de région sa double casquette ${ }^{125}$ ?». La consécration d'un préfet de région à plein temps serait la suite logique de l'ensemble des évolutions qui ont permis son affirmation progressive.

Les raisons qui justifient l'incohérence du dédoublement fonctionnel soulèvent également la question de la pertinence de l'unité de statut.

122. Christophe Guettier, op. cit., p. 422.

123. Jean-Louis Dufeigneux, «Le préfet de Région et l'interministérialité: les relations avec les services déconcentrés ", Administration, ${ }^{\circ}{ }^{179}$, octobre 1998, p. 58-60.

124. D. $n^{\circ} 85-779,24$ juill. 1985 , art. $1^{\mathrm{er}}$.

125. Jean-Michel Bricault, "Régionalisation de l'administration déconcentrée...», art. cité, p. 1997. 


\subsection{La remise en cause de l'unité de statut}

En plus du dédoublement fonctionnel, le décret du 29 avril 2004 modifié consacre une unité du statut préfectoral. Ses dispositions générales et communes s'appliquent indifféremment au préfet de région et au préfet de département. Le statut préfectoral est régi par le décret $n^{0} 64-805 \mathrm{du}$ 29 juillet 1964 fixant les dispositions réglementaires applicables aux préfets. Les préfets de région et les préfets de département sont soumis aux mêmes conditions de nomination, ont les mêmes droits et obligations et respectent les mêmes conditions de cessation des fonctions.

Comme pour le dédoublement fonctionnel, la montée en puissance du préfet de région semble remettre en cause cette unité du statut préfectoral. Il ne convient plus de revenir sur les justifications de cette unité de statut et les raisons qui militeraient en faveur de sa remise en cause, mais d'évoquer directement les perspectives d'évolution du statut des deux types de préfets. Deux options sont envisageables : la solution de compromis, qui serait de faire évoluer le système actuel, et la solution extrême, qui serait l'éclatement du statut des deux autorités.

La première option consisterait à conserver le principe de l'unité de statut, moyennant des adaptations pour le rendre cohérent avec l'importance et la prééminence du préfet de région. Dans cette perspective, la nomination aux fonctions de préfet de région pourrait être considérée comme un élément de valorisation dans le déroulement de la carrière de préfet de département. Un tel système pourrait être appliqué aux autres services régionaux, comme le passage au SGAR avant l'accès au grade de préfet ${ }^{126}$. Cette option présente plusieurs avantages. Elle permet de conserver l'unité de statut, chère aux préfets de département, tout en faisant du préfet de région une autorité expérimentée dans le corps de préfet de département. Mais son principal inconvénient reste le maintien de l'unité de statut, qui pourrait ainsi réduire l'autorité du préfet de région.

La seconde option est l'éclatement pur et simple des deux statuts et la consécration d'un statut autonome de préfet de région. Cette option permettrait de renforcer la hiérarchie fonctionnelle du préfet de région par une hiérarchie statutaire, gage d'affirmation de son autorité. Bien qu'elle soit conforme à l'évolution des réformes administratives, sa mise en œuvre reste problématique. La méfiance des préfets de département à l'égard d'une telle solution susciterait une prudence des pouvoirs

126. Paul Bernard, «Le relais régional...», art. cité, p. 24. 
publics. Ainsi, la consécration d'un véritable statut de préfet de région ne paraît pas encore acquise.

L'analyse de la place du préfet de région révèle un contraste entre l'affirmation de sa fonction et les incohérences de son statut. Avec l'accroissement de ses compétences, il est devenu un agent stratégique dans l'organisation administrative. Les impératifs d'efficacité de l'action publique et de réduction des moyens risquent de renforcer davantage la régionalisation administrative et les pouvoirs des préfets de région. La principale question soulevée par la promotion du préfet de région est celle de l'unité du corps préfectoral, donc de la cohabitation et des rapports qu'il entretient avec le préfet de département ${ }^{127}$. Les inquiétudes exprimées sur la menace de la cohésion de l'État et la dilution de son autorité semblent en revanche marginales ${ }^{128}$, car le préfet de région reste lui-même soumis au pouvoir hiérarchique des ministres.

La fonction de préfet de région a atteint sa maturité, si bien que la question de son statut mériterait d'être tranchée. La solution retenue, consistant à lui reconnaître une autorité pour l'exercice des fonctions tout en maintenant l'unité de statut avec le préfet de département, ne paraît pas satisfaisante. Ce compromis, « sans être "boiteux" [ne semble] guère durable ${ }^{129}$. La question de l'éclatement du statut des deux préfets mériterait tôt ou tard d'être réglée. Il y va de l'implantation durable du préfet de région comme autorité territoriale de référence.

Docteur en droit public de l'université de Rouen Assistant à l'université Ouaga II (Burkina Faso)

127. La marche de l'administration préfectorale, op. cit., p. 89.

128. Jean-Pierre Raffarin, "La montée en puissance de la région», Administration, $\mathrm{n}^{\mathrm{o}} 179$, octobre 1998 , p. 28.

129. Jean-Marie Pontier, op. cit., p. 820. 